used the following questionnaires: Female Sexual Function Index (FSFI), Brief Index of Sexual Function for Women (BISF-W), Sexual Quality of Life Questionnaire (SQoL-F), Pelvic Organ Prolapse/Urinary Incontinence Sexual Questionnaire (PISQ-12), Pelvic Floor Distress Inventory Questionnaire (PFIQ7), Fatigue Impact Scale (FIS), Beck's Depression Inventory II (BDI II), Health Assessment Questionnaire (HAQ), Scleroderma Health Assessment Questionnaire (SHAQ) and Human Activity Profile (HAP).

Results Compared to HC, patients with SSc had significantly higher prevalence and greater severity of sexual dysfunction (FSFI, BISF-W: in all subscales as well as total scores), dysfunction of pelvic floor (PISQ-12, PFIQ7), and worse sexual quality of life (SQol-F) (table). Worse scores in SSc patients were associated with higher disease activity [ESSG activity index: SQol-F $(\mathrm{r}=-0.364, \mathrm{p}=0.0443)$ ], greater fatigue [FIS correlated negatively with both FSFI, and BISF-W], more severe depression [BDI-II: FSFI $(\mathrm{r}=-0.553, \mathrm{p}=0.0002), \quad$ BISF-W $\quad(\mathrm{r}=-0.514$, $\mathrm{p}=0.0007)$, deteriorated quality of life [SHAQ: FSFI $(\mathrm{r}=-0.536, \mathrm{p}=0.0003)$, BISF-W $(\mathrm{r}=-0.563, \mathrm{p}=0.0001)$, SQol$\mathrm{F}(\mathrm{r}=-0.338, \mathrm{p}=0.0382)$, PISQ-12 $(\mathrm{r}=0.563, \mathrm{p}=0.0051)]$, and worse ability to perform physical activities [HAP: FSFI $(r=0.407, p=0.0082)$, BISF-W $(r=0.409, p=0.0078)]$.

Conclusions Women with SSc reported significantly impaired sexual function, sexual quality of life and pelvic floor function than age-matched healthy controls. Worse scores in SSc were associated with disease activity, physical activity, fatigue, depression and quality of life.

Acknowledgements Supported by AZV-16-33574A, MHCR 023728, and SVV - 260373.

Disclosure of Interest None declared.

\section{P026 CONTROL OF A2,6-SIALYLATION IN B-CELLS, AND CONSEQUENCES OF REDUCED B-CELL SIALYLATION IN PATIENTS WITH RHEUMATOID ARTHRITIS}

'L Johnston*, 'L Ouboussad, ${ }^{1,2} \mathrm{~A}$ Aslam, ${ }^{1,2} \mathrm{MH}$ Buch. 'Lirmm, University of Leeds; ${ }^{2} \mathrm{NIHR}$ Leeds Biomedical Research Centre, Leeds Teaching Hospitals Trust, Leeds, UK

\subsection{6/annrheumdis-2018-EWRR2019.20}

Career situation of first and presenting author Student for a master or a PhD.

Introduction Sialic acids are a family of 9-carbon sugars, added to the termini of glycoprotein chains, which are present on the surface of many cells, and secreted proteins. ${ }^{1}$ Sialic acids on glycan chains of the Fc fragment of IgG molecules can affect how IgG binds Fc receptors. ${ }^{2}$ In RA and other autoimmune conditions, disease specific auto-antibodies display decreased Fc sialylation compared with normal $\mathrm{IgG}^{3}{ }^{3}$ It has also been shown that plasmablasts from patients with RA display reduced cell surface sialylation compared to healthy control cells. ${ }^{4}$ Factors which determine B-cell surface sialylation, including regulation of ST6Gal1and NEU1 which add and cleave sialic acid from glycoprotein chains respectively, and consequences of altered sialylation have not been well described.

Objectives The aim of this study was to investigate factors which influence $\alpha 2,6$-sialylation in B-cells from the peripheral blood of healthy controls and patients with RA, and to investigate regulation of expression of ST6Gal1 and NEU1 in Bcells.

Methods B-cells isolated from the peripheral blood of healthy volunteers were cultured for 48 hours in the presence of TLR ligands, anti-IgM, CD40L, cytokines (TNF, IL-4, IL-6, IL-17) before $\alpha 2,6$-sialylation of the B-cell surface, and mRNA expression of genes related to $\alpha 2,6$-sialylation were measured by SNA lectin flow cytometry, and qPCR respectively. PBMCs were also isolated from peripheral blood and $\mathrm{T}$ and $\mathrm{B}$-cells activated using $\mathrm{CD} 3 / 28$ beads and anti-IgM/CD40L respectively, before measuring B-cell sialylation and expression of ST6Gal1 and NEU1 mRNA.

Results Surface $\alpha 2,6$ sialylation was found to be increased in B-cells stimulated with TLR ligands or anti-IgM/CD40L. This was accompanied by a decrease in expression of both ST6Gal1 and NEU1 mRNA over 48 hours. Surface sialylation was also increased by activated T-cells in co-culture experiments, however sialylation was reduced in B-cell cultures in the presence of TNF.

Conclusions $\alpha 2,6$-sialic acid is upregulated on the surface of healthy control B-cells in response to activation in vitro, and may be downregulated by TNF. Further experiments will determine if the environment in which naïve/memory B-cells are activated determines plasmablast/plasma cell sialylation, and if B-cells from patients with RA undergo the same changes in response to stimuli.

\section{REFERENCES}

1. Traving C, Schauer R. Cell Mol Life Sci 1998;54:1330-1349.

2. Sondermann P, et al. PNAS 2013; 110:9868-9872.

3. Dekkers $\mathrm{G}$, et al. Front Immunol 2018;9:553.

4. Pfeifle R, et al. Nat Immunol 2017;18(1):104-113.

Disclosure of Interest None declared.

\section{P027 SRC-LIKE ADAPTOR PROTEIN EXPRESSION IN RHEUMATOID ARTHRITIS}

${ }^{1} \mathrm{P}$ Királyhidi*, ${ }^{1} \mathrm{ES}$ Molnár, ${ }^{1} \mathrm{E}$ B Baricza, ${ }^{1,2} \mathrm{~N}$ Marton, ${ }^{1} \mathrm{E}$ Buzás, ${ }^{1} \mathrm{G}$ Nagy. ${ }^{1}$ Department of Genetics, Cell- and Immunobiology, Semmelweis University; ${ }^{2} J a h n$ Ferenc South-Pest Hospital, Budapest, Hungary

\subsection{6/annrheumdis-2018-EWRR2019.21}

Career situation of first and presenting author Student for a master or a $\mathrm{PhD}$.

Introduction The Src-like adaptor protein (SLAP) plays a central role in the fine regulation of both B- and T-lymphocyte activation. According to our previuos data SLAP is responsible for the proteasomal degradation of the CD3 zeta chain (CD3 $)$ ) of T-lymphocytes. ${ }^{1}$

Objectives We studied the effect of IL-10, IL-17A and TNF- $\alpha$ treatment on the SLAP expression of $\mathrm{CD}^{+}{ }^{+} \mathrm{T}-$ and $\mathrm{CD} 19^{+} \mathrm{B}-$ lymphocytes.

Methods Peripherial mononuclear cells (PMBC) were isolated from healthy donors and rheumatoid arthritis (RA) patients. $\mathrm{CD}^{+}$T-cells or $\mathrm{CD} 19^{+}$B-cells were isolated by negative magnetic separation; stimulated with ConA and goat antihuman $\operatorname{IgG}+\operatorname{IgM} F\left(\mathrm{ab}^{\prime}\right) 2$ fragments respectively. The samples were treated with IL-17A $(20 \mathrm{ng} / \mathrm{ml}$ and $80 \mathrm{ng} / \mathrm{ml}, 24$ hour), IL-10 (100 U/ml, 48 hour and 72 hour $)$ and TNF- $\alpha(20 \mathrm{ng} / \mathrm{ml}$ and $60 \mathrm{ng} / \mathrm{ml}, 24$ hour). The SLAP and CD3 $\zeta$ expression were measured by Western blot.

Results Both the SLAP and the CD3 $\zeta$ expression of the MTX treated patients' CD4 cells were higher upon IL-17A and IL10 treatment, than those of the MTX non-treated RA patients' or healthy donors'. The TNF- $\alpha$ induced SLAP expression of RA patients' CD19 B-cells was higher than those of the healthy donors' $B$ cells $(p=0.05)$. 
Conclusions In addition to proinflammatory and anti-inflammatory cytokines MTX treatment regulates the SLAP and the CD3 $\zeta$ expression of human T-cells.

\section{REFERENCE}

1. Érsek B, et al. CD3 $\zeta$-Chain Expression of Human T Lymphocytes Is Regulated by TNF via Src-like Adaptor Protein-Dependent Proteasomal Degradation. J Immunol. 2012.

Acknowledgements OTKA K77537.

Disclosure of Interest None declared.

\section{\begin{tabular}{|l|l} 
P030 & TRANSCRIPTIONAL LANDSCAPES OF MEMORY T CELLS
\end{tabular} FROM PATIENTS WITH JUVENILE IDIOPATHIC ARTHRITIS}

${ }^{1} \mathrm{P}$ Maschmeyer, ${ }^{1} \mathrm{GA}$ Heinz, ${ }^{1} \mathrm{~F}$ Heinrich, ${ }^{1} \mathrm{P}$ Durek, ${ }^{1} \mathrm{LE}$ Wirth, ${ }^{2} \mathrm{SL}$ von Stuckrad, ${ }^{1} \mathrm{~K}$ Lehmann, ${ }^{1} \mathrm{CL}$ Tran, ${ }^{2} \mathrm{~B}$ Orak, ${ }^{1} \mathrm{H}-\mathrm{D}$ Chang, ${ }^{1,2} \mathrm{~T}$ Kallinich, ${ }^{1} \mathrm{~A}$ Radbruch, ${ }^{1} \mathrm{M}-$ F Mashreghi*. 'German Rheumatism Research Centre (DRFZ); ${ }^{2}$ Charite University Medicine, Berlin, Germany

\subsection{6/annrheumdis-2018-EWRR2019.22}

Career situation of first and presenting author Post-doctoral fellow.

Introduction Juvenile idiopathic arthritis (JIA) is a chronic inflammatory disease (CID) of unknown origin and is characterized by joint inflammation in children and young adults. ${ }^{1}$ Evidence suggests a strong contribution of memory $\mathrm{T}$ cells to disease pathogenicity in JIA. While few markers for $\mathrm{T}$ cells adapted to chronic inflammation have been identified. ${ }^{2}{ }^{3}$ a comprehensive analysis of what distinguishes pathogenic memory $\mathrm{T}$ cells in inflamed tissues of chronic inflammation from protective, circulating memory $\mathrm{T}$ cells is still lacking.

Objectives To characterize the transcriptional profiles of memory $\mathrm{T}$ cells that putatively maintain chronic inflammation in JIA patients.

To identify biomarkers that are associated with autoantigenspecific clonotypes among memory $\mathrm{T}$ cells in JIA.

Methods Memory $\mathrm{T}$ cells were isolated from the synovial fluid (SF) and the peripheral blood (PB) of oligoarticular JIA patients and purified by fluorescence-activated cell sorting (FACS). Subsequently, single cell sequencing including $\mathrm{T}$ cell receptor (TCR) sequencing was performed on $\sim 18.000$ memory $\mathrm{T}$ cells of each JIA patient.

Results Memory $\mathrm{T}$ cell populations both from the blood and from the SF are heterogenous populations according to their transcriptional expression patterns. The SF harbored a larger population of enriched $\mathrm{T}$ memory cell clonotypes than the blood. In addition, enriched memory $\mathrm{T}$ helper cell clones in the SF showed a transcriptional pattern of activation compared to non-enriched clonotypes. Finally, small subpopulations of enriched memory $\mathrm{T}$ helper cell clones in the SF show a transcriptional signature that resembles transcriptomes obtained by bulk sequencing. Thus, a rather small subpopulation of antigen-specific cells might be responsible for the overall transcriptional character of $\mathrm{T}$ cells found at the inflamed sites of CIDs.

Conclusions Single cell sequencing combined with TCR sequencing is a powerful tool to identify and characterize subsets of $\mathrm{T}$ memory cells in chronic inflammation. The obtained data might be useful to better understand how $\mathrm{T}$ cell subsets contribute to disease pathogenicity in CIDs and reveals putative targets that could be therapeutically exploited in order to selectively deplete pathogenic memory $\mathrm{T}$ cells.

\section{REFERENCES}

1. Prakken $B$, et al. Juvenile idiopathic arthritis. Lancet 2011.

2. Niesner $U$, et al. Autoregulation of Th1-mediated inflammation by twist1. J Exp Med 2008.

3. Maschmeyer $\mathrm{P}$, et al. Selective targeting of pro-inflammatory Th1 cells by microRNA-148a-specific antagomirs in vivo. J Autoimmun 2018.

Acknowledgements This work is supported by the European Regional Development Fund (ERDF 2014-2020 and EFRE 1.8/11).

Disclosure of Interest None declared.

\section{P031/012 SIALIC ACIDS NEGATIVELY AFFECT THE BONE RESORPTIVE CAPACITY OF OSTEOCLASTS}

${ }^{1}$ AK Molhoek*, ${ }^{1}$ RE Li, ${ }^{2}$ ID Jansen, ${ }^{2} \mathrm{~T}$ Schoenmaker, ${ }^{2} \mathrm{TJ}$ de Vries, ${ }^{1} \mathrm{SJ}$ van Vliet, ${ }^{1} \mathrm{Y}$ van Kooyk. 'Molecular Cell Biology and Immunology, Amsterdam UMC; ${ }^{2}$ Periodontology, Academic Center for Dentistry, Amsterdam, Netherlands

\subsection{6/annrheumdis-2018-EWRR2019.23}

Career situation of first and presenting author Student for a master or a $\mathrm{PhD}$.

Introduction In rheumatoid arthritis the imbalance between osteoblasts and osteoclasts leads to an increased bone resorption, resulting in extensive bone destruction. Osteoclasts are multinucleated cells formed after fusion of macrophages. Macrophages are known to acquire an anti-inflammatory phenotype upon engagement of the carbohydrate sialic acid ${ }^{1}$, however whether triggering by sialic acids affects osteoclastogenesis is unknown. Therefore, we investigated the effect of sialic acids on osteoclastogenesis and their expression of the sialic acid binding receptors, called Siglecs.

Methods Human monocytes were plated on either plastic, glass or bone slices and stimulated with M-CSF and RANK-L to generate osteoclasts. We designed a novel sorting methodology to separate the osteoclasts and the residual macrophages in our osteoclastderived cultures. Purified osteoclasts were reanalyzed by microscopy and assessed for gene expression of osteoclast-specific genes by RT-PCR. Flow cytometry was used to obtain a full expression profile of the different siglec receptors in the osteoclasts, macrophages and monocytes precursor cells. Stimulation of pre-osteoclasts with sialic acids was done at day 7 and 10 of the culture. Supernatants were assessed for IL-6 and IL-10 production.

Results Our novel osteoclast purification method resulted in pure osteoclasts expressing the classical genetic osteoclast markers such as cathepsin $\mathrm{K}$ and TRAP, while being negative for CD14. During the transition to osteoclasts, expression of siglec-1, $-5 / 14$ and -7 was lost, while siglec-6, -9 and -15 expression was maintained or gained. Osteoclasts are known for their effectively bone resorption, however after adding sialic acids to the pre-osteoclasts, both osteoclast formation and bone resorption were reduced, while an increase of IL-6 and IL-10 cytokines was observed.

Conclusions Pre-osteoclasts stimulated with sialic acids display reduced osteoclast formation and bone resorption. Based on the siglec profiling we hypothesize that sialic acids engage siglec-9 on osteoclasts. Macrophages stimulated via siglec- 9 produce IL-10 cytokine, ${ }^{1}$ similarly as we observed in our stimulation experiments. Currently, we are addressing the role of siglec-9 in osteoclastogenesis.

\section{REFERENCE}

1. Ando $M$, et al. Siglec-9 enhances IL-10 production in macrophages via tyrosinebased motifs. Biochem Biophys Res Commun 2008;369(3):878-83.

Disclosure of Interest None declared. 